

\section{SOBRE LO OBJETIVO, LO SUBJETIVO Y LO CAPRICHOSO EN ARQUITECTURA} ON THE OBJECTIVE, THE SUBJECTIVE AND THE CAPRICIOUS IN ARCHITECTURE

Francisco Javier Montero Fernández

SUMMARY A new issue of a magazine must contemplate an editorial, as a project needs to be born of reasoning and a first sketch as steps towards offering a final image. We will keep the editorial in a drawer and develop the issue of the magazine. Within a few months, we will take out that sketch and be able to recognize its effectiveness; it will undergo some adjustment but we hope that it is still valid.

A journey begins with an undertaking, a destination and a purpose. Each issue of this journal presents a polyhedral character, demarcating the definition of Architecture facet by facet. Whenever we speak of Architecture we try to understand it, to delimit it and there are those who think that the secret of something unknowable could be enclosed within its anatomy. Step by step we slowly approach its understanding, thanks to an effort similar to that made by Sisyphus. It would be close to think of our approach as similar to the route of a Möbius strip, and although we can complete the route, we will always be conscious of the short and intangible distance existing at the same point on each side of the strip. This journey, which many want to understand as absurd, is the journey of the subjective, of that which allows us to approach ideas to understand them little by little, slowly, as opposed to the effective Iogical, analytical, anatomical knowledge that some researchers try to apply, like the child who takes things apart, disassembling the buildings thinking that the ideas are within. Despite all, they are forced to recognize that this work is absurd because it leads us to nothing, perhaps only to the destruction of thought in favour of the ineffective knowledge of the reality.

The other understanding that we propose consists of an alternative attitude, that which restores the subjective over the objective, to take note of the world that surrounds us, of the subjects that interest us, of universal ideas in order to establish a mechanism of knowledge, so that each one establishes creative short circuits that allow us to advance. Nobody must confuse the subjective with the capricious, with the absurdly brilliant or falsely artistic.

Successively, the journeys and the evolution of architectural ideas offered in this issue attempt an approach that demands an active atitude on the part of the reader so that, if they wish, they can assume certain ideas that may arise as references or guidelines to follow in their creative work.. 
n número nuevo de una revista debe pensar una editorial, al igual que un proyecto necesita nacer de un argumento y un primer croquis para, inicialmente, ofrecer una imagen finalista. Después guardaremos la editorial en un cajón y desarrollaremos el numero de la revista. Dentro de unos meses, sacaremos ese croquis y podremos reconocer su eficacia; sufrirá algún retoque pero esperamos que aún sea válido.

Un viaje comienza con un empeño, un destino o una finalidad. Esta revista se ofrece en cada número con un carácter poliédrico acotando faceta a faceta, la definición de Arquitectura. Siempre que hablamos de ella intentamos conocerla, delimitarla y hay quien piensa que en su anatomía puede encerrarse el secreto de algo que es inaprensible. Paso a paso nos acercamos a su conocimiento lentamente, gracias a un esfuerzo parecido al del trabajo de Sísifo. Lo más acertado sería pensar que nuestro acercamiento es parecido al recorrido de una cinta de Moebius y aunque consigamos agotar el recorrido, siempre seremos conscientes de la breve e inasible distancia existente en el mismo punto de cada lado de la cinta. Este viaje que muchos quieren entender como absurdo, es el viaje de lo subjetivo, de aquello que nos permite acercarnos a las ideas para conocerlas poco a poco, lentamente, frente al eficaz conocimiento lógico, analítico, anatómico que, como el niño que desmonta los objetos, algunos investigadores intentan aplicar, desmontando los edificios, pensando que dentro están las ideas. No obstante todos se ven obligados a reconocer que ese trabajo sí que es absurdo porque nos lleva a la nada, quizás solo a la destrucción del pensamiento a favor del conocimiento ineficaz de la realidad.

El otro conocimiento que proponemos consiste en una actitud alternativa, aquella que reivindica lo subjetivo frente a lo objetivo, para establecer como mecanismo de conocimiento una acotación del mundo que nos rodea, de los temas que nos interesan, de las ideas universales, para que cada uno establezca cortocircuitos creativos que nos permitan avanzar. Nadie debe confundir lo subjetivo con lo caprichoso, con lo absurdamente genial o lo falsamente artístico.

Sucesivamente los viajes y las traslaciones que se ofrecen en este número, intentan un acercamiento que exige una actitud activa por parte del lector, de manera que si quiere pueda asumir ciertas ideas que se dan a conocer como acotación o pautas a seguir en el trabajo creativo a desarrollar por cada uno.

El pintor František Kupka inició un viaje vital a inicios del siglo XX desde su Bohemia natal a París, pasando por Viena. Su pintura no objetiva evolucionó hasta que en 1912 los parisinos pudieron observar la primera exposición abstracta del autor, mucho antes de que otros autores como Kandisnky, Delauny, Malévitch o Mondrian. Una geografía recorrida e intelectualizada llenó su obra de decoraciones geométricas que fundamentaron su abstracción, permitiendo mantener el componente decorativo dentro del racionalismo más radical. 
El viaje convertido en mecanismo de formación y fuente de inspiración alcanza el referente más claro para la arquitectura en el siglo XX, con los viajes de Le Corbusier, el cual, en sus primeros itinerarios (viaje a la Toscana), valora la observación directa y la atención a temas decorativos con una absoluta desnudez de lo cotidiano, en una exaltación de la arquitectura eterna, alejada de la contaminación de su presente y en la búsqueda de la ornamentación como dato anatómico del conocimiento de la Arquitectura. Años después un ornamento mucho más abstracto cubrirá los mosaicos de las puertas de sus edificios, los grandes murales, los tapices y pinturas del autor.

En una Europa llena de nacionalidades, origen de tantos conflictos, el viaje se convierte en un inevitable complemento de formación gracias a la descontextualización, lo que permitió que, en casos como el sueco, la formación de sus artistas se completara en Roma hasta 1848 y, posteriormente en Dusseldorf o Munich para finalizar en París. En Asplund observaremos la influencia del Mediterráneo, en su interés por las superficies, el color y la dualidad exterior-interior, frente al paisaje y tratamiento de la luz nórdica. Su obra asumirá los conceptos aprendidos y nos dejará ejemplos como el Cine Skandia con su cielo lleno de elementos colgantes, la Biblioteca Nacional de estucos negros con claras referencias egipcias y griegas o el Cementerio del Bosque, donde las luces místicas del bosque se prolongan al interior de la Capilla, o la casa de verano en Stennäs. En este momento, el racionalismo aporta una conciencia vital a una Europa necesitada de encontrar referencias no caducas y la obra de Asplund ofrecía no sólo edificios sino la creación de un mundo en el que se superponían de manera constante el valor de lo local, de lo innato, con el de lo aprendido en el viaje.

Frente al escenario vacío, racional y perfectamente ajustado, Hugo Häring nos ofrece la aportación de lo cotidiano a través de la idea del espacio orgánico como el lugar donde se produce el acontecer de la vida. Frente al escenario geométrico, Häring pone en valor el espacio sobre la forma y la realidad, vista desde este prisma, se nos vuelve diferente. Los objetos dejan de ser una forma evolucionada para convertirse en la respuesta a una función o al comportamiento de una herramienta. Los edificios articulan una orientación, construyen una curva de nivel, organizan un horario o articulan la privacidad, hasta convertirse en prótesis de uso.

De manera nada casual surge el debate entre ingeniería y arquitectura personalizado en autores como Owen Williams que nos lleva hasta más allá de la mitad del siglo XX, habiéndose convertido en un autor de referencia en cualquier tema, obviando el debate entre tradicionalismo y modernidad, hasta el extremo de inscribirse como arquitecto en 1930 después de haber ejercido como ingeniero. Obras como la sede del Daily Express marcarán una referencia fuera del anterior debate abriendo nuevos caminos a la valoración de la arquitectura, abandonando la decoración que revestía edificios convirtiéndolos en tramoyas formales. Sus obras finales miran a las grandes infraestructuras viales confirmando el devenir personal constante entre arquitectura e ingeniería como un viaje de identidad de Williams que nos deja excelentes ejemplos sobre los que reflexionar la diferencia entre invención y creación.

En este devenir surgen análisis transversales que permiten conocer un determinado hito en su contexto, el tiempo se vuelve transversal y analizamos la importancia de los hechos en relación a otros. El final de los años 30 del siglo XX, solo puede ser estudiado de esta manera, con 
el conocimiento paralelo de otros acontecimientos, de manera que la simultaneidad convierte este momento en un tiempo barroco. No podemos mirar hacia un punto, sino que cualquier hecho debe ser analizado desde su simultaneidad frente a otros hitos que ocurren a la vez, y el mundo, se nos hace extenso de manera diversa, no solo por la dimensión física sino por complejidad temporal. Richard Buckminster Fuller nos mostrará su primera propuesta de vivienda "Dimaxion" en 1927 -a la vez que se construyen las obras más representativas de la arquitectura moderna- y 20 años después retomará este experimento persiguiendo la posibilidad de la prefabricación aplicando los medios de la industria del automóvil o la aviación. Fuller vivió un periodo importante en el Black Mountain College y se relaciona con tres episodios, fundamentalmente vinculados con la construcción de una cúpula geodésica, como una forma alternativa de habitación, de protección de las actividades del hombre. Un lugar sin materia, una estricta envolvente, puro espacio interior inhabitable y hermético. Los años de Buckminster Fuller en Black Mountain se aproximan a la aparición de los happenings, a la consideración de que la obra de arte es un acontecimiento y como tal debemos entender la construcción de la primera cúpula geodésica con estudiantes en la puesta en valor de la simultaneidad de actuaciones, ideas y contextos.

Uno de los hitos de la arquitectura del siglo XX es la construcción de dos ciudades, Chandigarh y Brasilia. El tercer viaje de Le Corbusier a Chandigarh de los veintitrés que realizó a la India, se relaciona con la definición del Capitolio y se observa la distancia de edad y madurez respecto al precoz viaje a la Toscana. En los veinticinco días de este viaje surge el interés por las enormes distancias del país, sus llanuras, el cielo y los horizontes montañosos; la arquitectura se vuelve una interpretación del lugar y, a su vez, el viaje se vuelve creativo, abandonando el carácter estudioso del viaje a la Toscana. Conocer se convierte en origen de la creación a través de la interpretación y la solución final viaja en la mente del autor ofreciendo variaciones sobre un mismo tema de tal manera que la formalización final no es sino una de las posibilidades de un mismo proyecto.

La última pauta que ofrecemos mira al futuro como inevitable obsesión humana. El valor del tiempo es asumido a través de la consideración del futuro, de la capacidad potencial de avance y evolución del tiempo no vivido. La Ciencia Ficción es la herramienta para pensar en el futuro y el viaje es la relación espacio/temporal básica. Los escenarios propuestos mezclan la posibilidad con la realidad cotidiana en una mezcla sugerente y propositiva bañada de un posibilismo imaginativo. La imaginación debe gobernar el tinte de la realidad/ficción. Cartografías de la imaginación, mapas de viajes que nos muestran una realidad no real, o quizás demasiado real, pero en cualquier caso una realidad no inerte sino intencionada, una realidad de autor que nos muestra e interpreta ese tiempo y lugar de ficción que colorea nuestras mentes. La ciudad del futuro, el transporte; los viajes necesitan ser materializados en la visualización de escenarios que exige el cine.

Es una mirada ilusionada a un futuro que constantemente es superado por una realidad frenada por la incapacidad de otros sectores de la actividad humana, fundamentalmente políticos y económicos, que nos lastran hundiéndonos en la actual crisis. Esperemos que la libertad de la imaginación nos permita sobrevivir. 